\title{
Quarter-sweep arithmetic mean iterative algorithm to solve fourth-order parabolic equations
}

\begin{abstract}
The main purpose of this paper is to investigate the effectiveness of the Quarter-Sweep Arithmetic Mean (QSAM) iterative method by using the implicit finite difference approximation in order to solve fourth-order parabolic equations. The basic idea and the formulation of this method are also presented. In fact, implementations of Full-Sweep, HalfSweep, and Quarter-Sweep Arithmetic Mean methods, namely FSAM, HSAM and QSAM respectively are also shown. Finally, through numerical results obtained, it has shown that the QSAM iterative method is superior as compared with the FSAM and HSAM methods.
\end{abstract}

Keyword: Quarter-sweep iteration; Weighted mean scheme; Implicit scheme; Fourth-order parabolic equation 